\title{
Synthesis and biochemical evaluation of GnRH-III-drugconjugates
}

\author{
Sabine Schuster ${ }^{1}$, Beáta Biri-Kovács ${ }^{1}$, Adina Borbély ${ }^{2}$, Norbert Sewald ${ }^{2}$, Ines Neundorf ${ }^{3}$, Cesare Gennari ${ }^{4}$, Gábor Mezó ${ }^{1}$ \\ ${ }^{1}$ Eötvös Lorand University, Faculty of Science, Institute of Chemistry, Research Group of Peptide Chemistry, Budapest, Hungary \\ ${ }^{2}$ University of Bielefeld, Department of Chemistry, Organic and Bioorganic Chemistry, Bielefeld, Germany \\ ${ }^{3}$ University of Cologne, Institute of Biochemistry, Cologne, Germany \\ ${ }^{4}$ Università degli Studi di Milano, Dipartimento di Chimica, Milano, Italy
}

https://doi.org/10.17952/35EPS.2018.080

\section{Introduction}

Gonadotropin releasing hormone-III(GnRH-III, $<$ EHWSHDWKPG-NH $2 ;<$ E - pyroglutamic acid, isolated from sea lamprey), a native isoform of the human peptide hormoneGnRH-I, represents a promising starting point for the development of efficient peptide-based drug delivery systems (DDS) for targeted cancer therapy. Beneficially, GnRH-III specifically binds to GnRH receptors on cancer cells without revealing a significant endocrine effect [1]. Due to that, a variety of GnRH-III-drugconjugates have been designed and characterized in our laboratories, in which the anthracycline daunorubicin (Dau) was linked to GnRH-III via oxime bond by insertion of an aminooxyacetyl moiety [1]. To achieve an improved antitumoractivity, we synthesized a set of oxime-linked GnRH-III-Dau conjugates containing different unnaturalamino acids within the sequence and studied their in vitro anticancer activity [2,3]. The best compoundswere chosen for further biochemical evaluation and as targeting moiety of novel drug conjugates containing a self-immolative p-aminobenzyloxycarbonyl (PABC) spacer between a cathepsin B cleavable dipeptide (Val-Ala or Val-Cit) and the drug Dau or paclitaxel (PTX). For comparative purpose, non-cleavable GnRH-III-drug conjugates were also synthesized. All bioconjugates have been analyzed for their in vitro cytostatic effect and the release of the drug was followed by lysosomal degradation studies in presence of rat liver lysosomal homogenate. Furthermore, the highly efficient drug candidate cryptophycin was conjugated to a GnRH-III homing device by using different cleavable and noncleavable linker systems and the corresponding in vitro antitumor activity of the compounds was determined.

\section{Results and Discussion}

\subsection{Oxime bond containing GnRH-III-daunorubicinconjugates}

All GnRH-III- $\left[{ }^{8} \operatorname{Lys}(\mathrm{Dau}=\mathrm{Aoa})\right]$ derivatives were prepared by Fmoc-SPPS, whereby Dau was conjugated in solution to an aminooxyacetic acid linker at the side chain of ${ }^{8} \mathrm{Lys}$ by formation of an oxime bond. The cytostatic effect was determined by Alamar blue ${ }^{\oplus}$ assay and compared to the control compoundsK1 and K2 (Scheme 1).

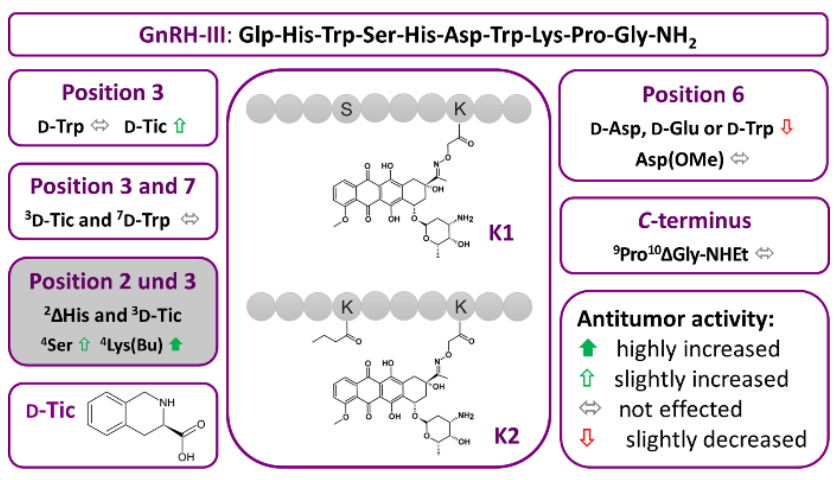

Scheme 1: Influence of sequence modification on the anticancer activity (on MCF-7 and HT-29 cancer cells) in comparison to the controls K1 and K2. D-Tic - (R)-1,2,3,4-tetrahydroisoquinoline-3-carboxylic acid.

All GnRH-III-Dau derivatives displayed an in vitro cytostatic effect on MCF-7 breast and HT-29 colon can-cer cells in a low micromolar range, whereby only compound P19-H (GnRH-III- $\left[{ }^{2} \Delta\right.$ His- $\left.{ }^{3} \mathrm{D}-\mathrm{Tic}-{ }^{4} \mathrm{Lys}(\mathrm{Bu})\right]$ $[8 \mathrm{Lys}(\mathrm{Dau}=\mathrm{Aoa})]$ ) displayed a highly improved antitumor activity on both human cancer cell lines (Figure 1A) $[2,3]$. To interpret these results, we performed additional studies of $\mathrm{P} 19-\mathrm{H}$ in direct comparison with our lead compound K2. Flow cytometry studies revealed an increased cellular uptake of compound P19-H on MCF7 (Figure 1B) and HT-29 cancer cells, whereby the uptake rate particularly at low compound concentrations was 
improved in comparison to K2. Additionally, receptor binding studies revealed similar binding affinities for both conjugates indicating that the receptor affinity is not retarded by amino acid substitution. Furthermore, we analyzed the release of the active drug metabolite in presence of lysosomal rat liver homogenate. The corresponding LC-MS data of $\mathrm{P} 19-\mathrm{H}$ and $\mathrm{K} 2$ showed that the $\mathrm{N}$-terminus of $\mathrm{P} 19-\mathrm{H}$ was more resistant to lysosomal enzymes. However, the smallest Dau-containing metabolite $\mathrm{H}-\mathrm{Lys}(\mathrm{Dau}=\mathrm{Aoa})-\mathrm{OH}$ was released within one hour in case of both conjugates. These outcomes confirmed that the release of the active metabolite is not decelerated by the $\mathrm{N}$-terminal modification of $\mathrm{P} 19-\mathrm{H}$, which is of great importance for the biological activity of the conjugate. Considering all findings, we assume that the improved anticancer activity of $\mathrm{P} 19-\mathrm{H}$ is mainly related to an enhanced cellular uptake [3].
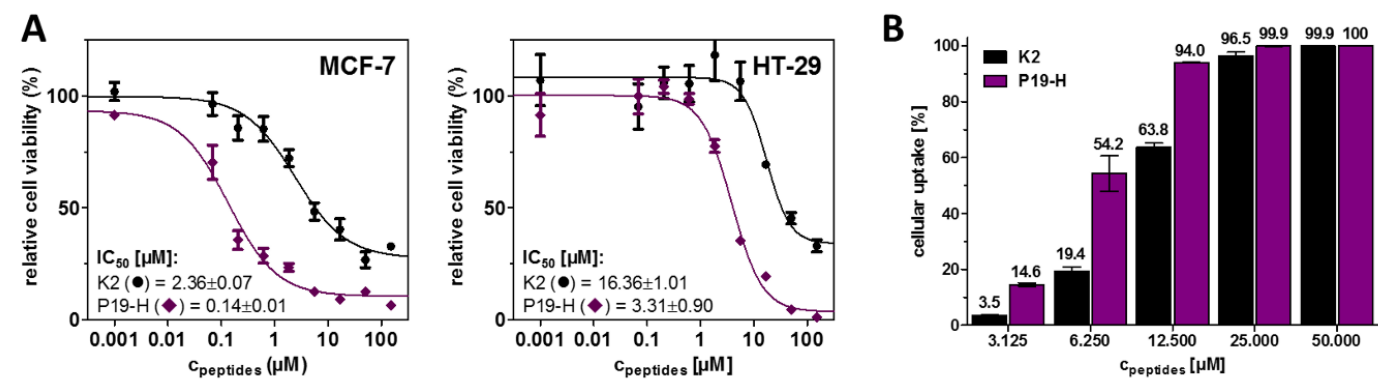

Figure 1: A) Anticancer activity of P19-H and K2 on MCF-7 and HT-29 human cancer cells after $24 \mathrm{~h}$ treatment and additional $48 \mathrm{~h}$ incubation. Curves obtained by non-linear regression (sigmoidal dose responds, $n=2$ ). B) Cellular uptake of the GnRH-III conjugates K2 and P19-H on MCF-7 cancer cells after $6 \mathrm{~h}$ treatment determined by flow cytometry. (Error bars represent standard deviation).

\subsection{Self-immolative linker containing GnRH-III-daunorubicirand paclitaxel conjugates}

Based on the promising results of K2 and P19-H, novel GnRH-III-PTX and Dau conjugates were developed by using the corresponding peptide sequence as targeting moiety. To ensure the release of the free drug, selfimmolative linker systems with cathepsin B cleavable sites (Val-Ala or Val-Cit) were applied to link the drugs. The peptide carriers were synthesized by Fmoc-SPPS, while the dipeptidyl-PABC-drug linkers, as well as the non-cleavable linkers were synthesized in solution as recently described $[4,5]$. The cytotoxic effect of the compounds was investigated on $\mathrm{GnRH}$-receptor positive A2780 ovarian carcinoma cells. The corresponding $\mathrm{IC}_{50}$ values of the Dau-conjugates emphasize that the novel targeting moiety (GnRH-III- $\left[{ }^{2} \Delta \mathrm{His}-{ }^{3} \mathrm{D}-\mathrm{Tic}-{ }^{4} \mathrm{Lys}(\mathrm{Bu})\right]$ has a beneficial impact on the antitumor activity with $\mathrm{I}_{50}$ values 2.5 -times lower than that of the GnRH-III$\left[{ }^{2} \mathrm{His}^{3}{ }^{3} \mathrm{Trp}^{-}{ }^{4} \mathrm{Lys}(\mathrm{Bu})\right]$ conjugates. Moreover, the outcomes indicate that the Val-Ala linker containing conjugates provide a slightly improved cytotoxic effect in comparison to the Val-Cit containing conjugates. This might be mainly related to the accelerated release of Dau. The correspondinglysosomal degradation studies pointed out that the Val-Ala spacer is cleaved already within the first 5 minutes, while the cleavage of the Val-Cit linker and release of free Dau was detected after one hour of incubation with rat liver lysosomal homogenate. On the contrary, the release of the free drug could not be detected in case of the non-cleavable linker containing conjugates, even after 24 hours incubation with lysosomal enzymes. Thus, it can be assumed that the decreased biological activity of these conjugates is related to the fact that the free Dau is not released, as well as to the fact that a modification of the amino group of the sugar moiety can lead to the loss of bioavailability [6]. Recent studies demonstrated a markedly decreased in vitro antitumor activity of GnRH-III conjugates by using the amino function of the daunosamine sugar for amide bond formation to a glutaryl-spacer [7].

Apart from that, the $\mathrm{IC}_{50}$ values of the PTX-conjugates display a similar anticancer activity for all cleavable conjugates which might be related to the releasing mechanism of the PTX. It has been reported that by using the PABC spacer in combination with the diamine cyclization linker, at first a diamine l inker containing prodrug is released. The following cyclisation of the diamine-linker to 1,3-dimethyl-2-imidazolidinone and the corresponding release of PTX has been reported to be the rate-limiting step of the self-immolative process $[4,8]$. Taking this into account, it can be assumed that the stability of the prodrug and the release of the free PTX has a higher impact on the antitumor activity of the GnRH-III conjugates than the targeting sequence or the cathepsin cleavable dipeptide spacer. In contrast, the non-cleavable GnRH-III-PTX conjugates displayed a highly reduced cytotoxic effect on GnRH-receptor positive A2780 ovarian cancer cells which clearly demonstrates that the 
release of the cytotoxic payload is of high importance for the biological activity of the bioconjugates and mainly related to the dipeptidyl spacer in combination with the self-immolative linker system.
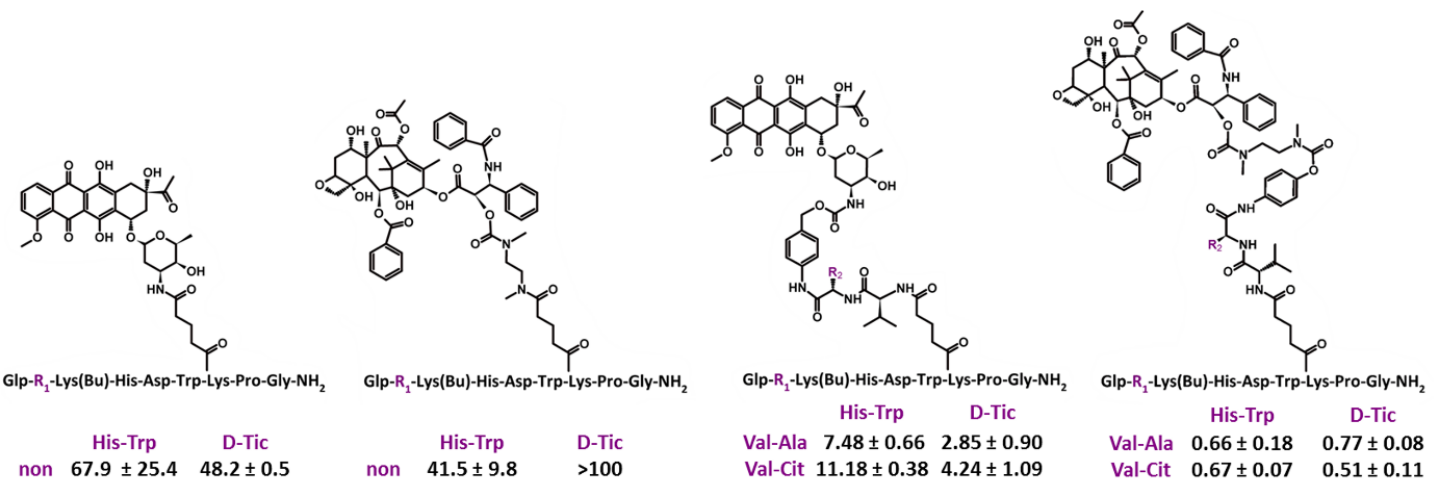

Figure 2: Structure of GnRH-III-Dau and PTX-conjugates and corresponding IC50 values [ $\mu M]$ on A2780 ovarian cancer cells. IC50 values were determined after $6 h$ (PTX) or 24 h (Dau) treatment and additional $66 \mathrm{~h}$ or 48 incubation.

\subsection{Cathepsin cleavable and non-cleavable GnRH-III-cryptophycinconjugates}

The highly efficient cryptophycin analog cryptophycin-55-glycinate (Cry-55-Gly) was used for the development of a novel GnRH-III based drug delivery system. The antiproliferative activity of cryptophycins, is based on their ability to destabilize microtubules, whereby these highly cytotoxic cyclo-depsipeptides provide a much higher potency than other tubulin effectors like paclitaxel or vinblastine [9]. The functionalized Cry-55-glycinate, which is suitable for conjugation to peptide-based homing devices, was prepared by total chemical synthesis as recently reported [10]. Moreover, the Cry-55-glycinate was combined with three different alkyne functionalized linker systems to ligate the payload to an azide-containing GnRH-III targeting moiety by copper catalyzed azide-alkyne cycloaddition. The designed linker systems contain either a non-cleavable spacer or a cathepsincleavable Arg-Asp-Arg-Val-Cit peptide spacer either in combination with the PABC self-immolative moiety or without. The resulting GnRH-III-Cry-55-Gly conjugates were analyzed for their cytotoxic activity on GnRHreceptor positive A2780 ovarian carcinoma cells (Figure 3). The resulting $\mathrm{IC}_{50}$ values of all three conjugates are in a low nanomolar range indicating that the linker structure did not substantially affect the biological activity of the compounds. Due to these results, we assume that the activity of the GnRH-III-Cry-55-Gly conjugates is not related to a cathepsin mediated drug release. A possible explanation might be that the conjugation of Cry-55-Gly to the peptide carrier did not affect the microtubule destabilization or the related biological activity of the drug.

In conclusion, all GnRH-III-Cry-55-Gly conjugates display highly efficient antiproliferative activity on GnRHreceptor positive A2780 ovarian cancer cells, whereby the biological activity was approximately 20-times higher in comparison to the related GnRH-III PTX conjugates. This emphasizes the potential of GnRH-III-cryptophycin conjugates for targeted tumor therapy and encourage us to further investigate this approach.

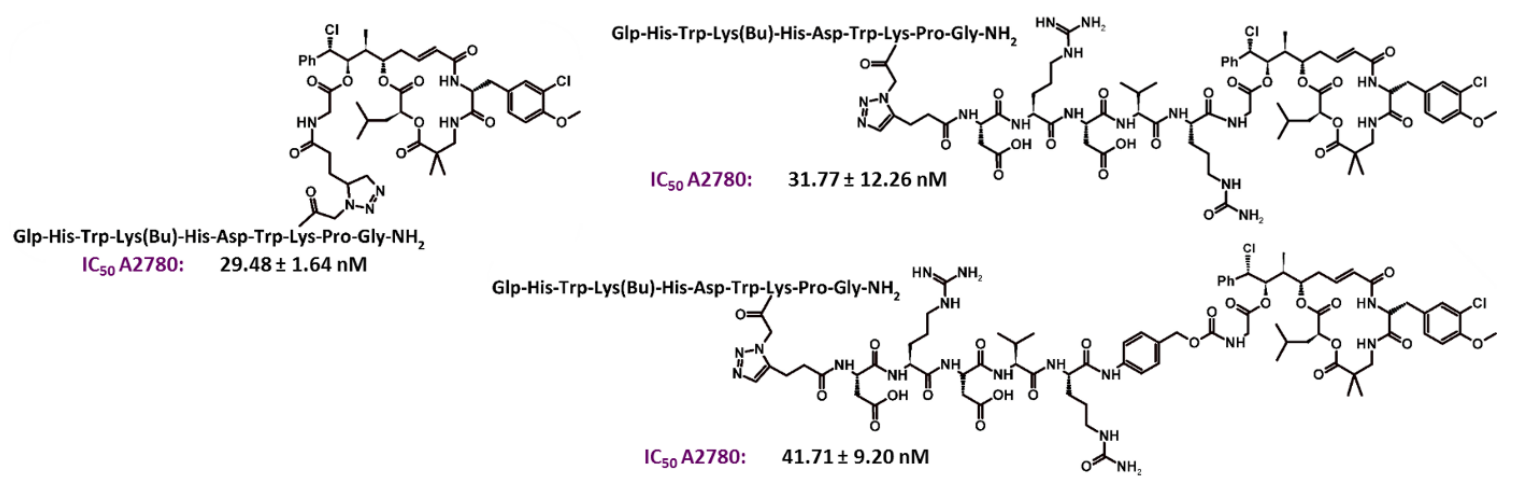

Figure 3: Structure of GnRH-III-Cryptophycin-conjugates and corresponding IC50 values. The cytotoxic effect was determined by cell-viability assay on A2780 ovarian cancer cells after $6 \mathrm{~h}$ treatment and additional $66 \mathrm{~h}$ incubation. 


\section{Conclusion}

To study the influence of sequence modification in GnRH-III on the efficiency of tumor targeting, we synthesized and characterized 20 novel GnRH-Dau conjugates. The results reveal one highly promising candidate with a substantially improved antitumor activity in comparison to our lead compound. Thus, this compound was chosen for further investigation in vivo on tumor bearing mice. Moreover, the two most potent targeting sequences were successfully used for the development of novel GnRH-III-Dauand PTX conjugates containing two different cathepsin B labile dipeptides and a self-immolative moiety. The results of the cell viability assay on A2780 ovarian cancer cells confirm the beneficial impact of the novel targeting sequence on the anticancer activity of GnRH-III conjugates. Furthermore, we developed and evaluated three different GnRH-III-based cryptophycin conjugates. It was shown that all conjugates display $\mathrm{IC}_{50}$ values in a low nanomolar range, which further emphasizes the high potential of GnRH-III based drug conjugates for targeted tumor therapy.

This project has received funding from the European Union's Horizon 2020 research and innovation program under the Marie Sklodowska-Curie grant agreement No 642004, and from the National Research, Development and Innovation Office (NKFIH K119552 and NVKP_16-1-2016-0036), Hungary.

\section{References}

[1] G. Mezó, et al., 2007, Peptides, 28, 806-820

[2] S. Schuster et al., 2018, Beilstein J. Org. Chem., 14, 756-771

[3] S. Schuster et al., 2018, Pharmaceutics (under revision)

[4] A. Dal Corso et al., 2015, Chem. Eur. J., 21, 6921-6929

[5] G.M. Dubrowchik et al., 2002, Bioconjugate Chem., 13, 855-869

[6] Y-G. Gao et al., 1991, Proc. Natl. Acad, Sci. USA, 88, 4845-4849

[7] G. Mezó, et al., 2008, Current Medicinal Chemistry, 15, 2366-2379

[8] F.M.H. de Groot et al., 2001, J. Org. Chem., 66, 8815-8830

[9] C. Weiss et al., 2017, J. Pept Sci., 23, 514-531

[10] Cazzamalli et al., 2018, ACS Omega (accepted) 